Supplementary Information for:

Efficient and Stable Low-Bandgap Perovskite Solar Cells Enabled by a CsPbBr3Cluster Assisted Bottom-up Crystallization Approach

Liqiang Xie, Kebin Lin, Jianxun Lu, Wenjing Feng, Peiquan Song, Chuanzhong Yan, Kaikai Liu, Lina Shen, Chengbo Tian, and Zhanhua Wei*

Engineering Research Center of Environmental-Friendly Functional Materials, Ministry of Education, College of Materials Science \& Engineering, Huaqiao University, Xiamen 361021, China.

Correspondence and requests for materials should be addressed to Z.H. W. (email: weizhanhua@hqu.edu.cn) 


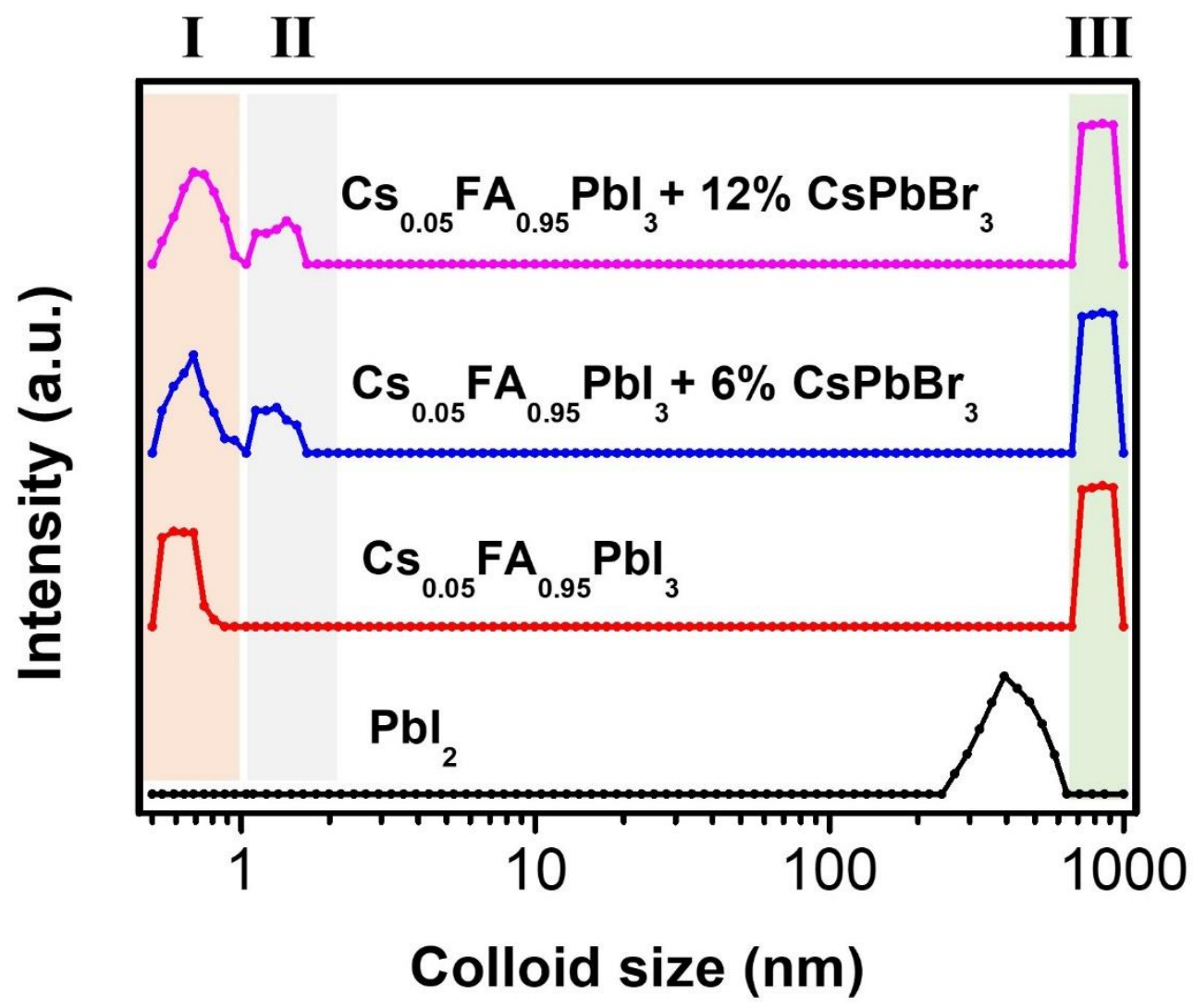

Supplementary Figure 1. $\mathrm{CsPbBr}_{3}$ effect on the colloidal chemistry of perovskite precursors investigated by DLS. Region I indicates the individual $\mathrm{Pb}-\mathrm{I}$ octahedron, region II indicates the $\mathrm{CsPbBr}_{3}$ cluster and region III indicates the soft coordination framework of precursors. 


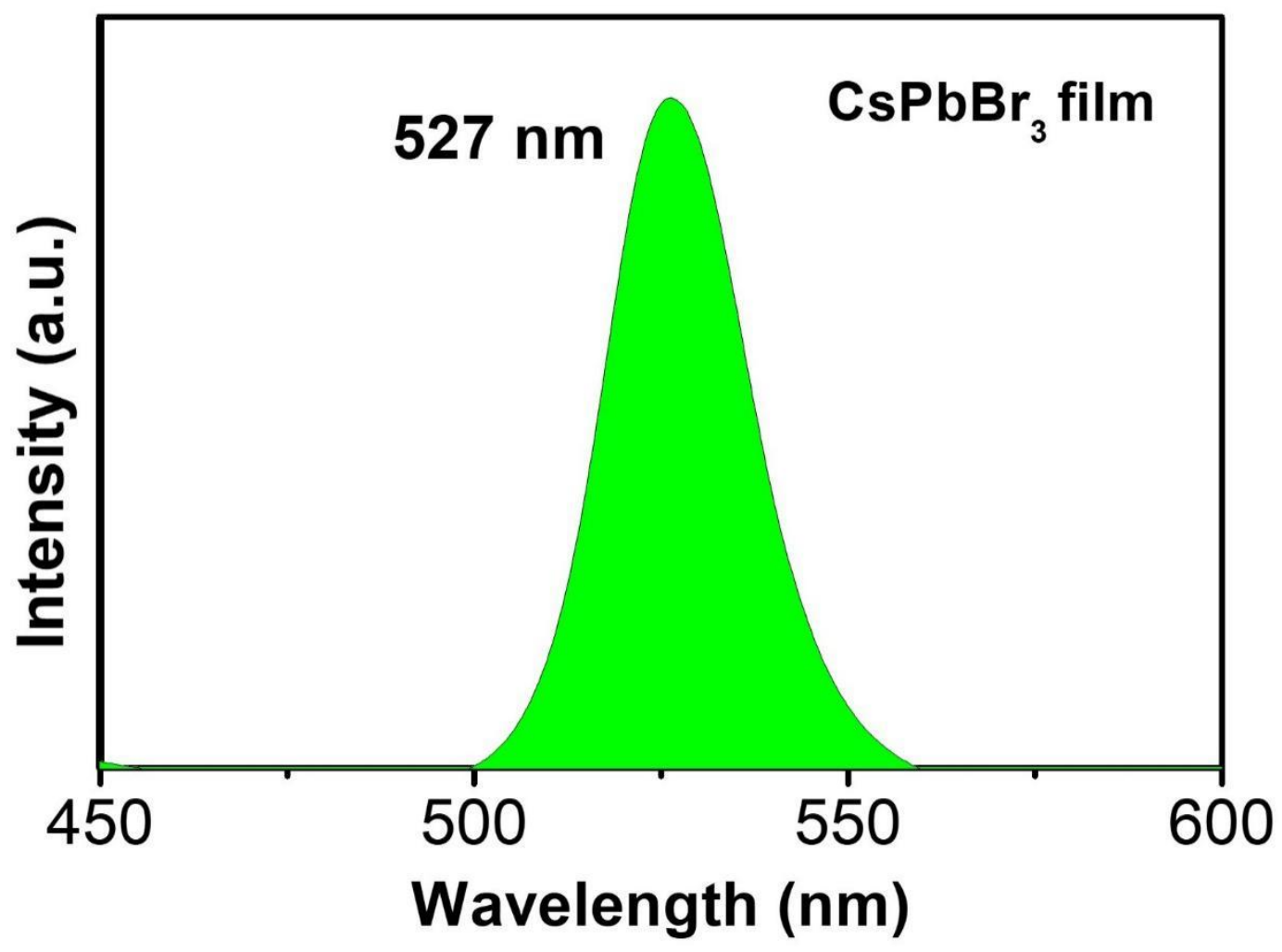

Supplementary Figure 2. Photoluminescence spectrum of $\mathrm{CsPbBr} 3$ film. 

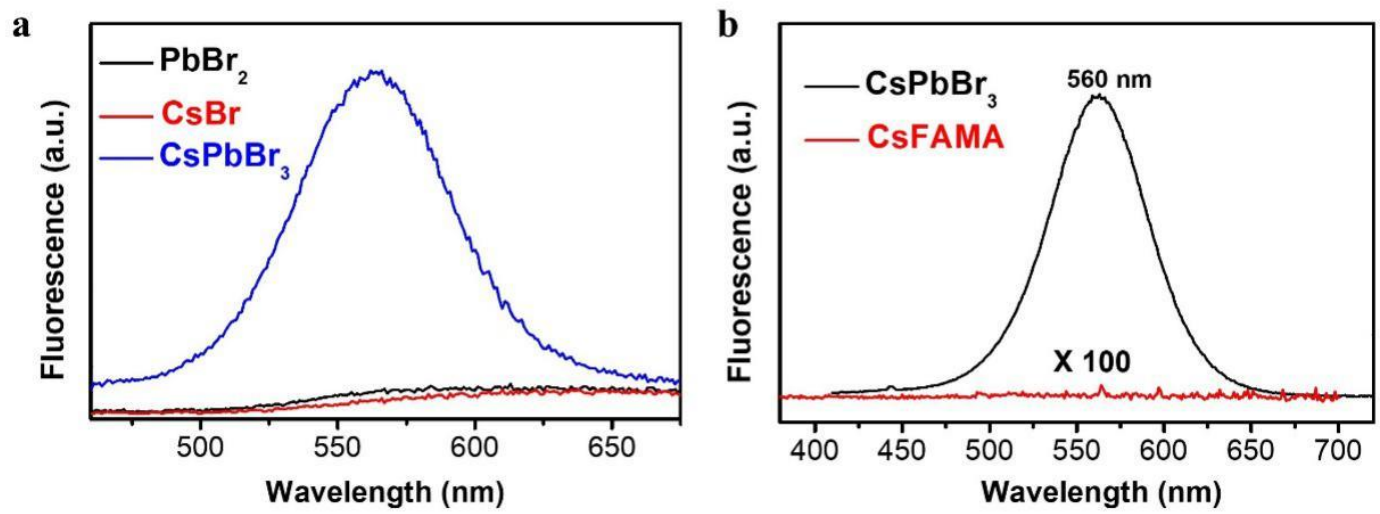

Supplementary Figure 3. a Fluorescence spectra of $\mathrm{PbBr}_{2}, \mathrm{CsBr}$ and $\mathrm{CsPbBr}_{3}$ dissolved in DMSO. b Fluorescence spectra of $\mathrm{CsPbBr}_{3}$ and CsFAMA triple-cation perovskite precursor solution. 
$\mathbf{a}$

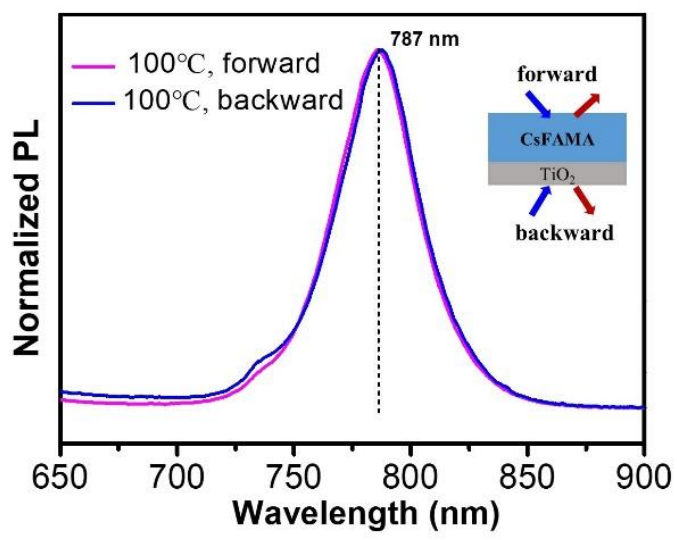

b

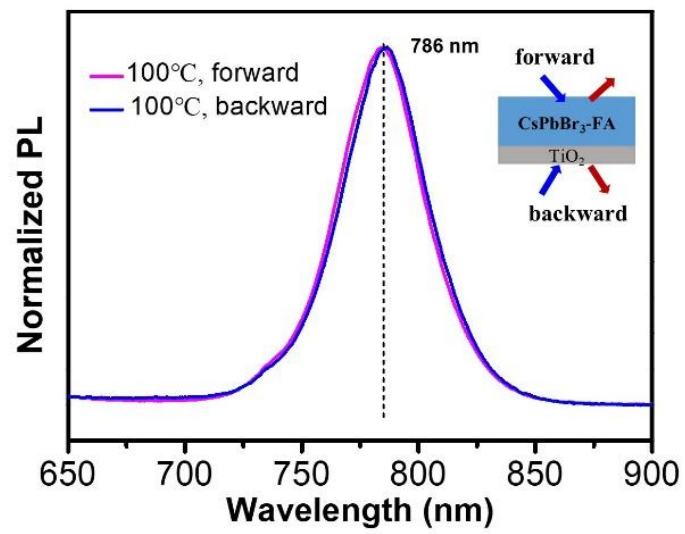

Supplementary Figure 4. Photoluminescence spectra of $100{ }^{\circ} \mathrm{C}$ annealed a CsFAMA and $\mathbf{b} \mathrm{CsPbBr}_{3}-\mathrm{FA}$ perovskite films, respectively. 


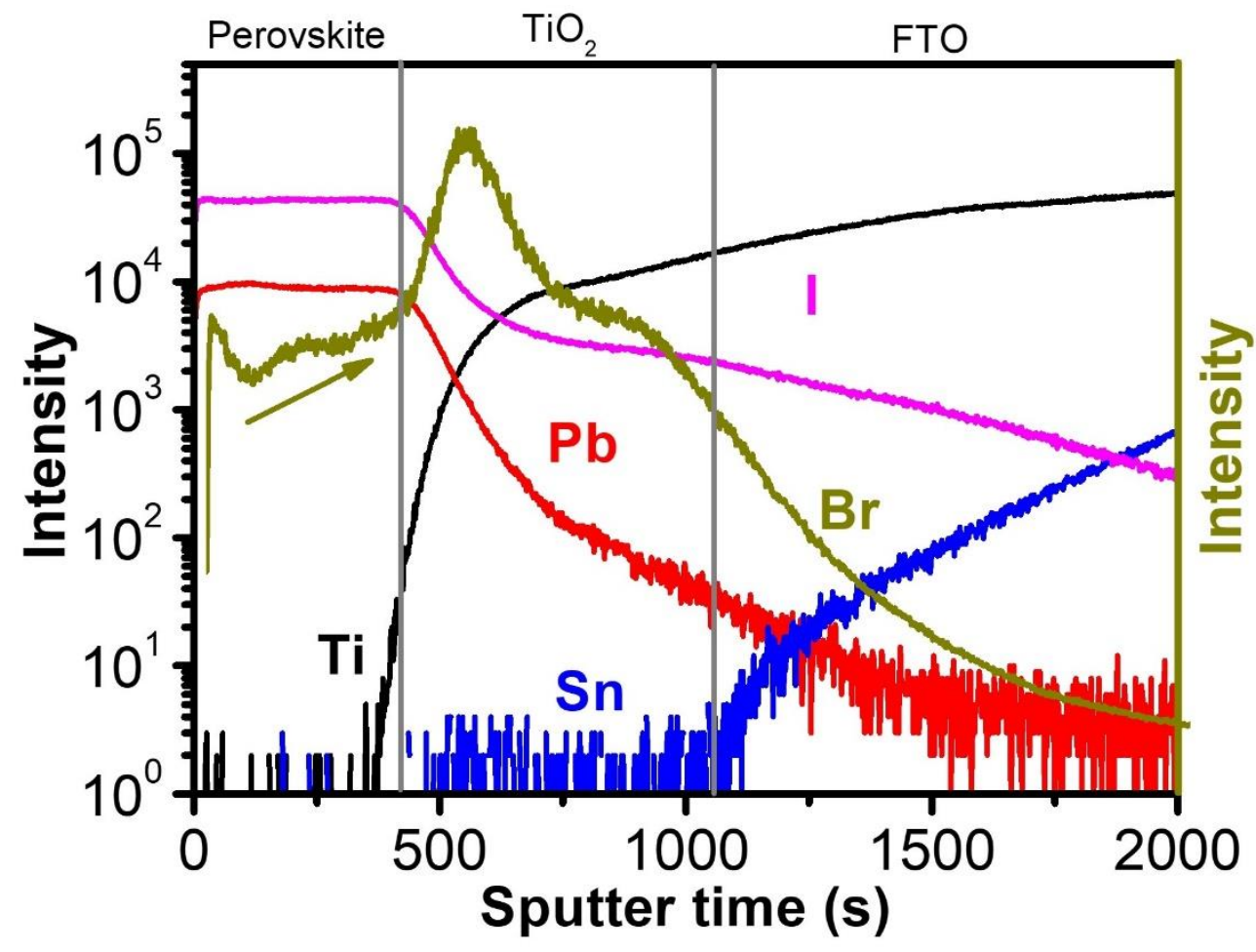

Supplementary Figure 5. ToF-SIMS depth profile analysis of a $\mathrm{CsPbBr}_{3}-\mathrm{FA}$ perovskite intermediate sample spin-coated at $25{ }^{\circ} \mathrm{C}$ in $\mathrm{N}_{2}$-filled glovebox without further thermal annealing. 

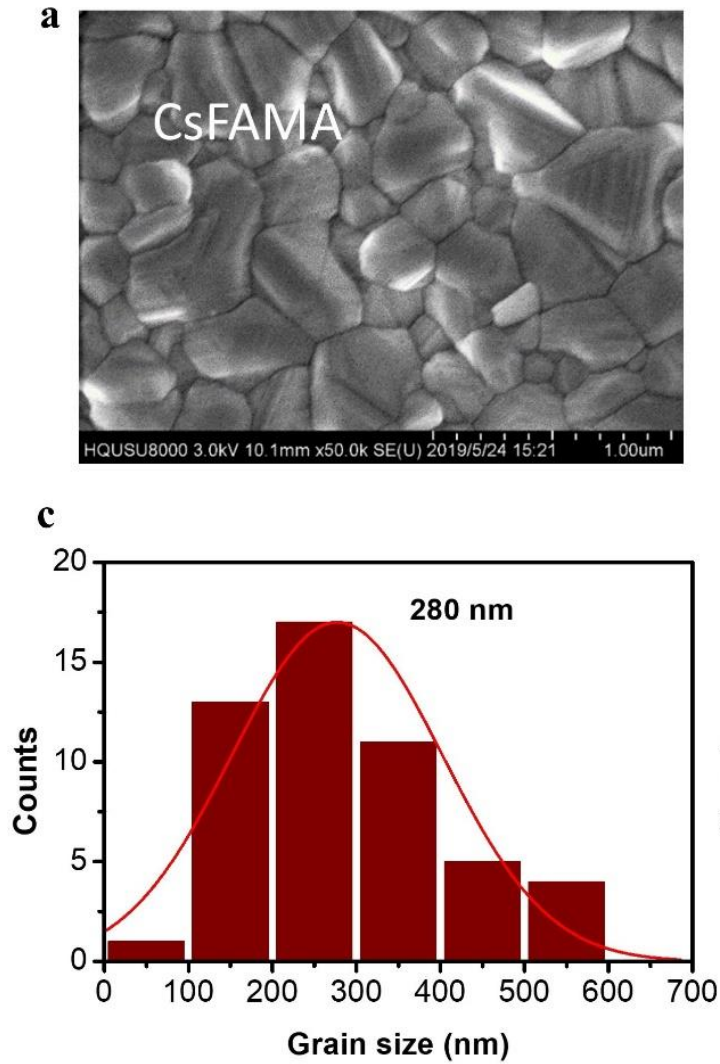

b

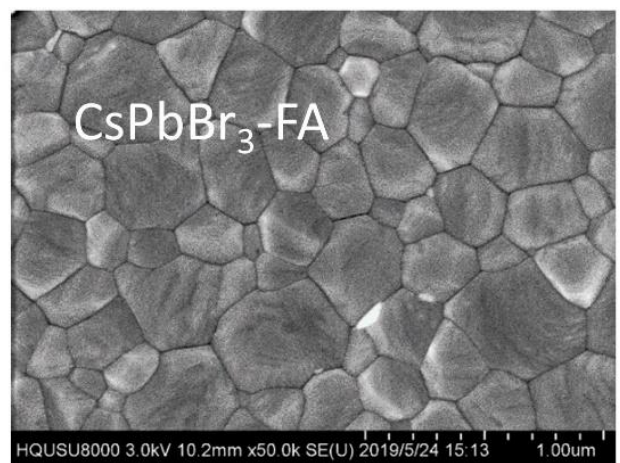

d

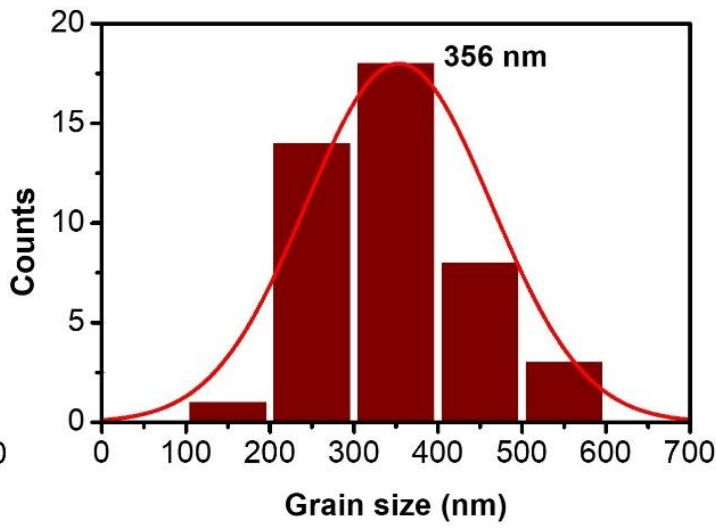

Supplementary Figure 6. SEM images and the corresponding grain size distribution for a c CsFAMA, b d $\mathrm{CsPbBr}_{3}-\mathrm{FA}$ perovskite films, respectively. 

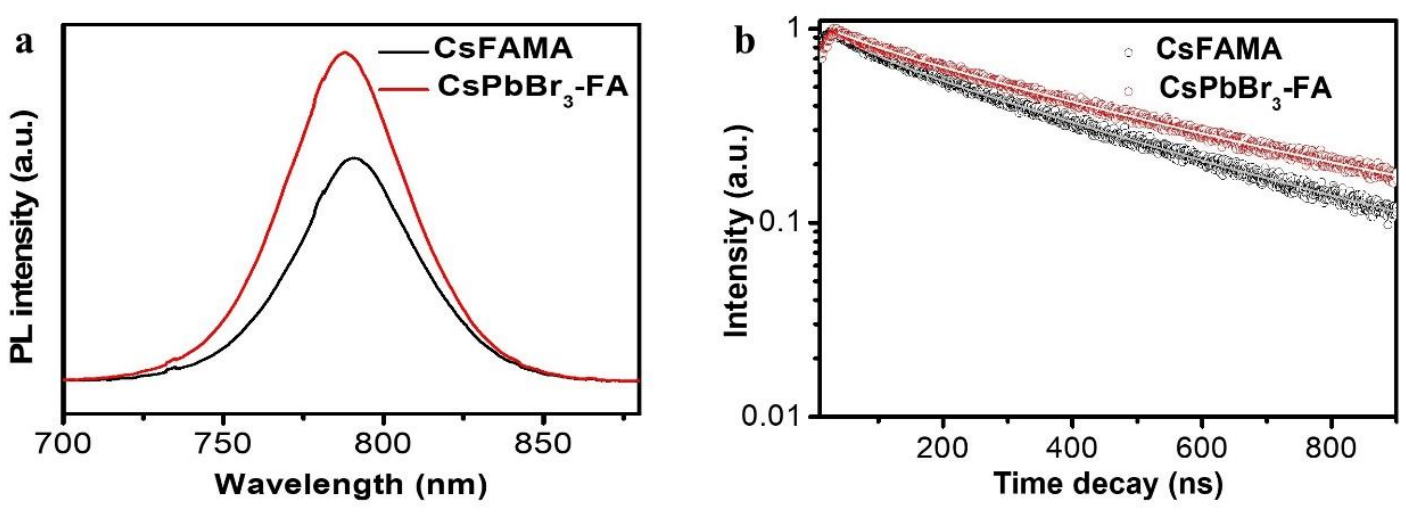

c

\begin{tabular}{cccccc}
\hline & $\boldsymbol{\tau}_{\mathbf{1}}$ (ns) & $\mathbf{A}_{\mathbf{1}}$ & $\boldsymbol{\tau}_{\mathbf{2}}$ (ns) & $\mathbf{A}_{\mathbf{2}}$ & $\boldsymbol{\chi}^{\mathbf{2}}$ \\
\hline CsFAMA & 62.04 & 0.253 & 388.24 & 0.831 & 0.997 \\
$\mathrm{CsPbBr}_{3}-\mathrm{FA}$ & 80.72 & 0.145 & 452.32 & 0.881 & 0.996 \\
\hline
\end{tabular}

Supplementary Figure 7. a Steady-state photoluminescence and $\mathbf{b}$ transient PL decay curves of the annealed $\left(100{ }^{\circ} \mathrm{C}\right)$ perovskite films for CsFAMA and $\mathrm{CsPbBr}_{3}-\mathrm{FA}$, respectively. c Fitting parameters of the TRPL results. 

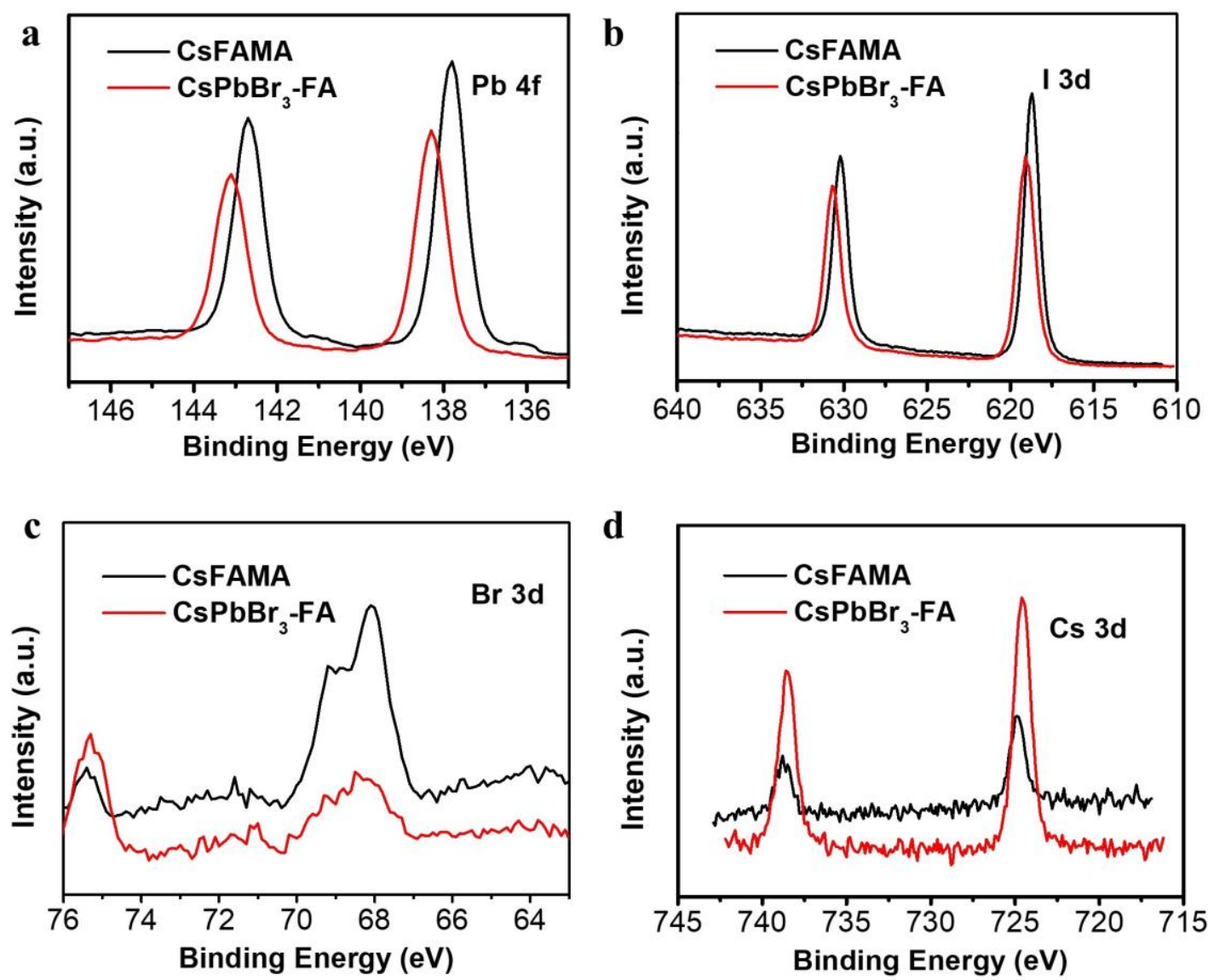

Supplementary Figure 8. XPS spectra of the as-prepared perovskite films. a $\mathrm{Pb} 4 \mathrm{f}, \mathbf{b}$

I $3 d, c$ Br $3 d$ and $\mathbf{d}$ Cs $3 d$. 
Supplementary Table 1. Photovoltaics parameters summaries of $\mathrm{CsPbBr}_{3}-\mathrm{FA}$ perovskite solar cells with varied molar concentration $(0 \%$ to $12 \%)$ of $\mathrm{CsPbBr}_{3}$ clusters.

\begin{tabular}{ccccc}
\hline $\begin{array}{c}\text { CsPbBr } \\
\text { molar ratio }\end{array}$ & $\begin{array}{c}\boldsymbol{J}_{S C} \\
\left(\mathbf{m A} / \mathbf{c m}^{2}\right)\end{array}$ & $\boldsymbol{V}_{\text {oC }}(\mathrm{V})$ & FF & PCE (\%) \\
\hline $0 \%$ & 25.20 & 0.994 & 0.736 & 18.42 \\
$1 \%$ & 24.72 & 1.033 & 0.750 & 19.45 \\
$2 \%$ & 24.66 & 1.082 & 0.766 & 20.43 \\
$4 \%$ & 24.42 & 1.098 & 0.773 & 20.72 \\
$6 \%$ & 24.71 & 1.096 & 0.776 & 21.02 \\
$9 \%$ & 24.30 & 1.062 & 0.734 & 18.94 \\
$12 \%$ & 23.00 & 1.066 & 0.738 & 18.11 \\
\hline
\end{tabular}


a

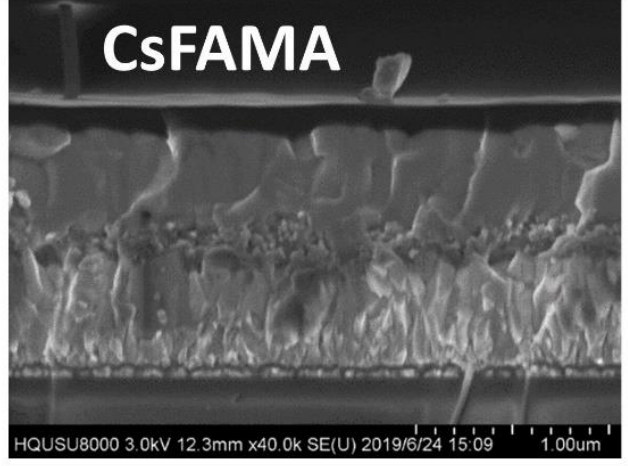

b

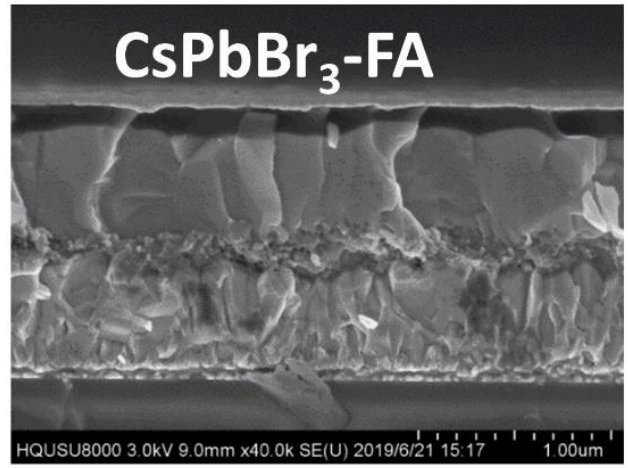

Supplementary Figure 9. Cross-sectional SEM images of a CsFAMA and $\mathbf{b} \mathrm{CsPbBr}_{3}-$

FA perovskite solar cells. 


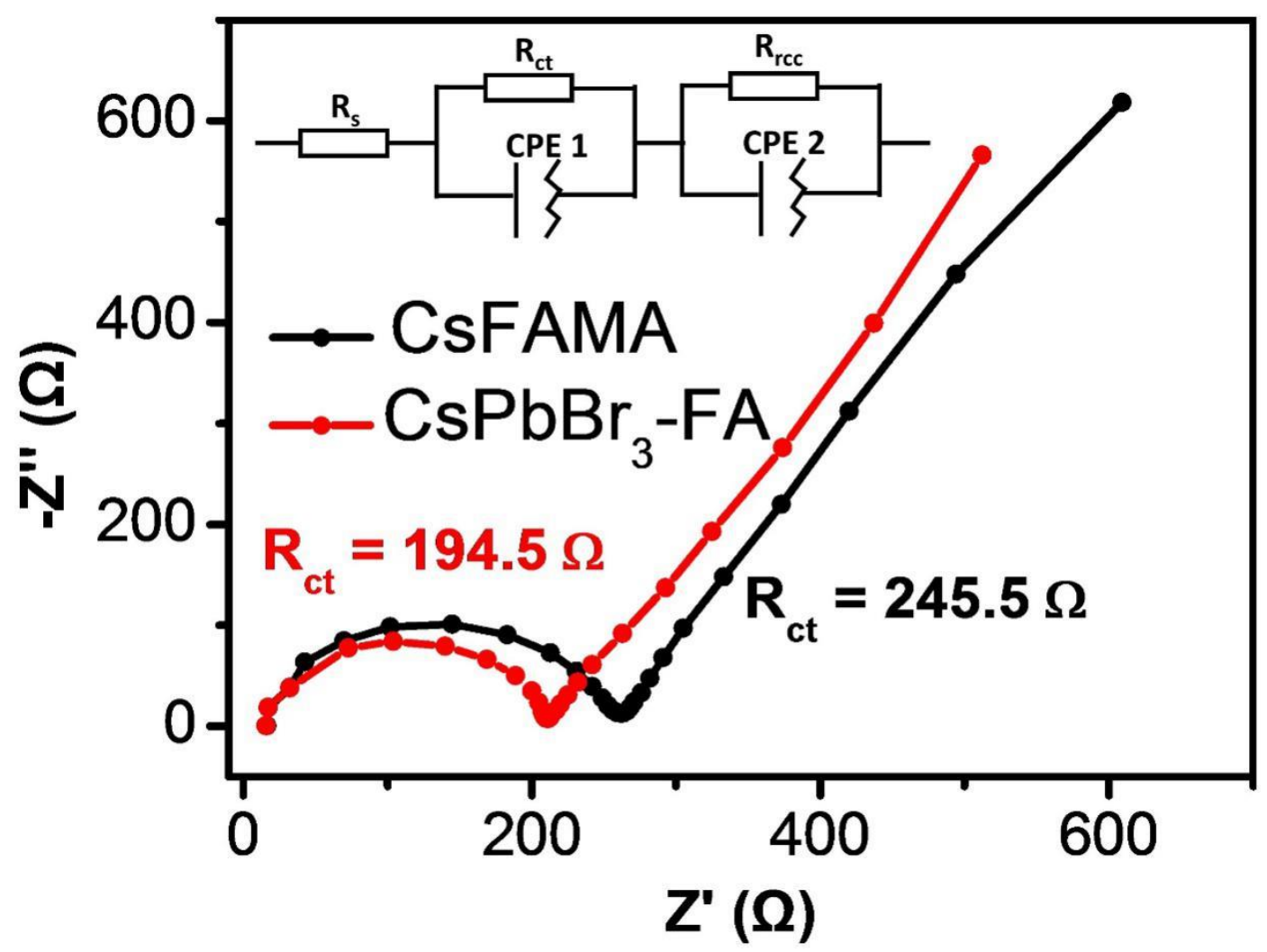

Supplementary Figure 10. Electrochemical impedance spectroscopy (EIS) of CsFAMA and $\mathrm{CsPbBr}_{3}-\mathrm{FA}$ perovskite solar cells measured at short circuit under $\mathrm{AM}$ $1.5 \mathrm{G}$ illumination. 


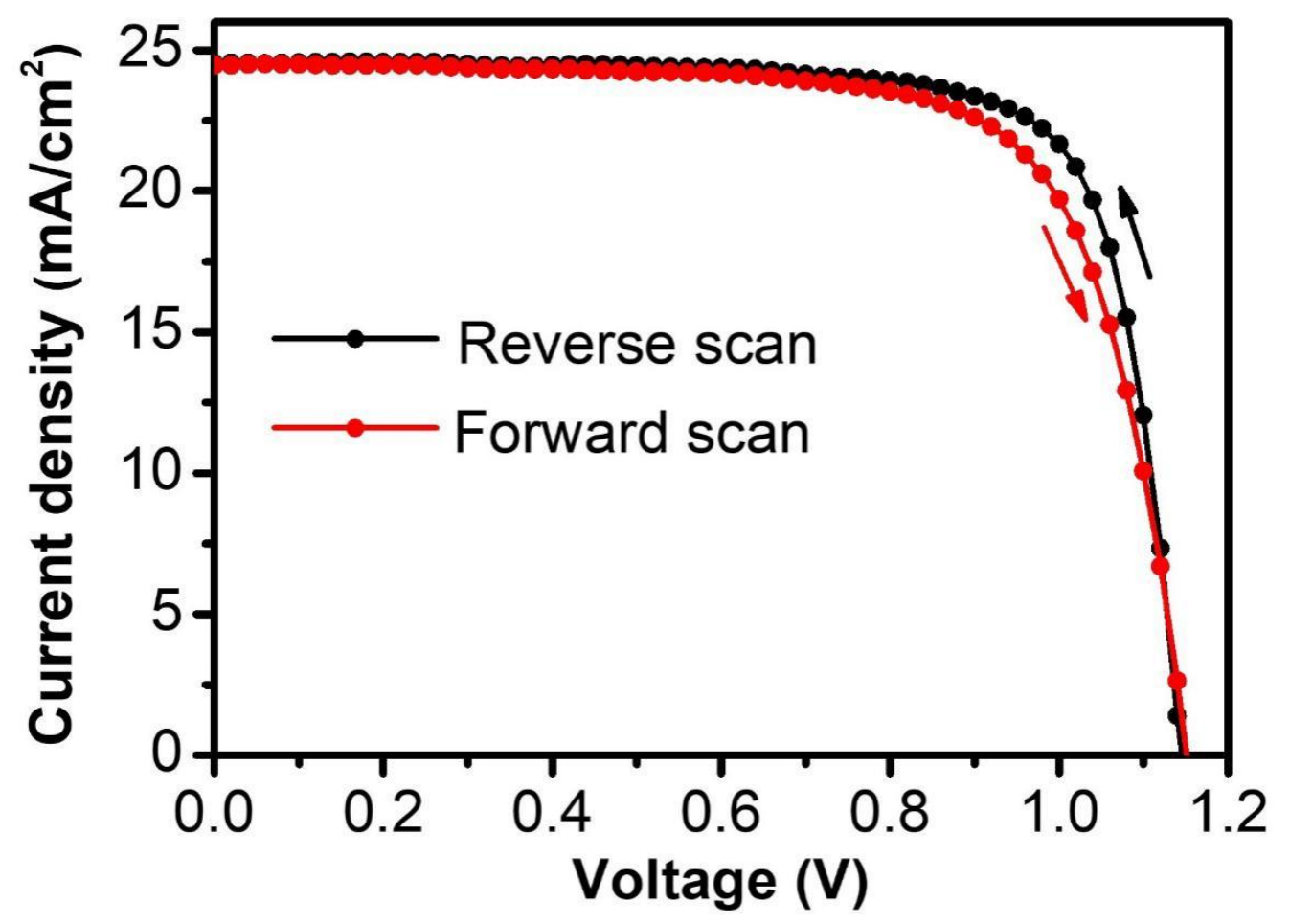

Supplementary Figure 11. $J-V$ curves of the $\mathrm{CsPbBr}_{3}-\mathrm{FA}$ champion device measured in the forward $(0 \mathrm{~V}$ to $1.2 \mathrm{~V})$ and reverse $(1.2 \mathrm{~V}$ to $0 \mathrm{~V})$ scanning directions. 
Supplementary Table 2. Photovoltaics parameters of the $\mathrm{CsPbBr}_{3}-\mathrm{FA}$ champion device using 6\% $\mathrm{CsPbBr}_{3}$ clusters.

\begin{tabular}{ccccc}
\hline & $\boldsymbol{J}_{S C}$ & $\boldsymbol{V}_{\boldsymbol{O C}}$ & Fill factor & PCE \\
\hline Reverse scan & 24.52 & 1.145 & 0.775 & 21.78 \\
Forward scan & 24.47 & 1.151 & 0.729 & 20.54 \\
Average & 24.49 & 1.148 & 0.752 & 21.16 \\
\hline
\end{tabular}




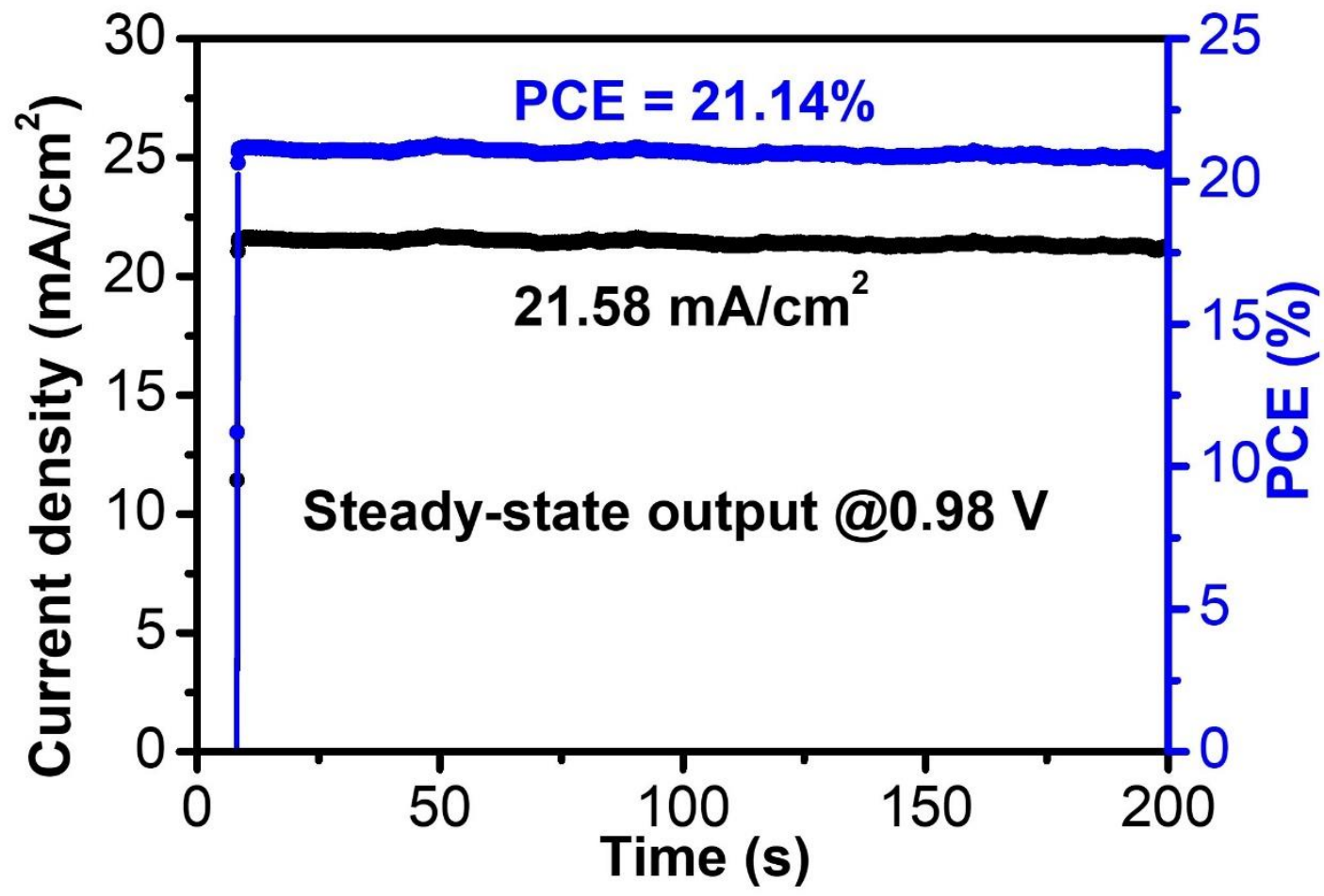

Supplementary Figure 12. Steady-state output of $\mathrm{CsPbBr}_{3}-\mathrm{FA}$ perovskite solar cells at $0.98 \mathrm{~V}$ measured under AM1.5 G illumination. 


\section{$500 \mathrm{~nm}$}

Supplementary Figure 13. Cross-sectional TEM image of the $\mathrm{CsPbBr}_{3}-\mathrm{FA}$ champion device prepared by FIB etching. 


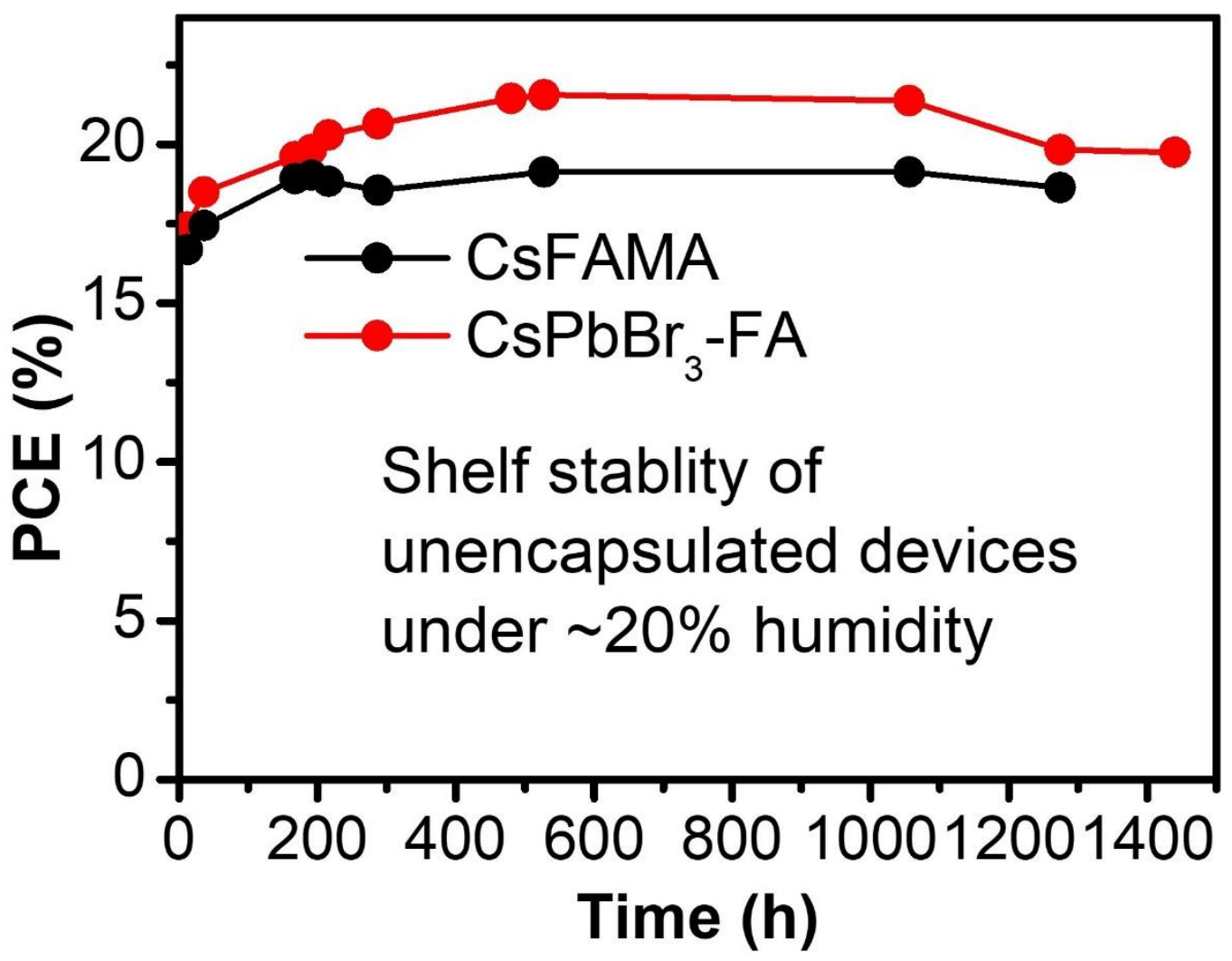

Supplementary Figure 14. Shelf stability of perovskite solar cells stored at room temperature and $20 \%$ relative humidity. 